INPLASY

PROTOCOL

To cite: Evans et al. Collecting linked patient reported and technology reported outcome measures for informing clinical decision making: a scoping review. Inplasy protocol

2021100038. doi:

10.37766/inplasy2021.10.0038

Received: 12 October 2021

Published: 12 October 2021

Corresponding author: lan Porter

i.porter@exeter.ac.uk

Author Affiliation:

University of Exeter

Support: University of Geneva/ Exeter.

Review Stage at time of this submission: Data extraction.

Conflicts of interest:

None declared.

\section{Collecting linked patient reported and technology reported outcome measures for informing clinical decision making: a scoping review}

Evans, J1; Porter, I2; Cockcroft, E³ Kassam, A4; Valderas, J5.

Review question / Objective: We aim to map out the existing research where concomitant use of patient reported and technology reported outcome measures is used for patients with musculoskeletal conditions.

Condition being studied: Musculoskeletal disorders (MSD) covering injuries or disorders of the muscles, nerves, tendons, joints, cartilage, and spinal discs. Musculoskeletal manifestations of joint pathology.

Eligibility criteria: 1) Peer-reviewed primary studies and literature reviews. Grey literature not included. 2) Studies which include co-administration of Patient-Reported Outcomes (PROMs) AND wearable electronic devices (e.g. fitness trackers, accelerometers, gyroscopes, pedometers smartphones, smartwatches) in musculoskeletal manifestations of joint pathology. Studies are EXCLUDED which feature wearable electronic devices but not concomitant/real time capturing of PROMs (e.g. they are recorded retrospectively/ at different timepoints). 3) Studies in languages other than English will be excluded unless a translation is available.

INPLASY registration number: This protocol was registered with the International Platform of Registered Systematic Review and Meta-Analysis Protocols (INPLASY) on 12 October 2021 and was last updated on 12 October 2021 (registration number INPLASY2021100038).

\section{INTRODUCTION}

Review question / Objective: We aim to map out the existing research where concomitant use of patient reported and technology reported outcome measures is used for patients with musculoskeletal conditions.
Rationale: Inactivity and poor self-care predispose older adults undergoing surgical interventions to decrease their long-term health and life quality outcomes. Currently, assessment of patients' physical and psychological states (e.g., activity) for a given short-term period (e.g., week) is 
conducted via momentary PatientReported Outcomes Measures (PROMs). PROMs suffer from reporting biases, ceiling, floor effects, and no sensitivity to change at their scale's extremes. Conversely, personal smartphones, and wearables are increasingly accurate in long-term behavioural TechnologyReported Outcomes (TechROs). However, the extent to which TechROs provide clinically useful information in the context of surgical interventions is unknown. PROMs and TechROs, including commercially available ones, have been widely used for people experiencing musculoskeletal manifestations, yet there has been limited research mapping the extent they have been used complimentarily.

Condition being studied: Musculoskeletal disorders (MSD) covering injuries or disorders of the muscles, nerves, tendons, joints, cartilage, and spinal discs. Musculoskeletal manifestations of joint pathology.

\section{METHODS}

Search strategy: The searches will be conducted in the following databases: MEDLINE (In-Process \& Other Non-Indexed Citations and Ovid MEDLINE (R), 1948 to present, accessed through OvidSP); Embase (1974 to present, accessed through OvidSP); PsycINFO (1967 to present, accessed through OvidSP); and CINAHL (from 1981 to present, accessed through EBSCO). The Cochrane Database of Systematic Reviews (CDSR) will also be searched. Searches will be conducted in English and studies in languages other than English will be excluded unless a translation is available. The following search terms will be used adapted to the specific requirements of each database, through the use of different thesaurus terms where applicable and truncation and wildcard characters. "PROM OR "PROMS" OR "patient reported outcome*" OR "patient reported assessment" OR "patient reported symptom"” "technology reported outcome ${ }^{\star ”}$ OR "digital health technology" OR "wearable electronic devices" OR "fitness trackers" OR "wear activity trackers" OR "wear electronic device" OR "wear diagnostic device" OR "wear computer device" OR "activity tracker" OR "accelerometry"OR "accelerometer" OR "gyroscope" OR "smart watch" OR "pedometer" Two reviewers will independently assess each reference in title and abstract form to assess whether eligibility criteria have been met. The eligibility criteria will be piloted with a random sample of 20 of all the studies/ documents downloaded. All the studies will be screened by the reviewers independently of each other. The same strategy will be adopted for the full-text documents selected for inclusion in this review. Only studies considered relevant by both reviewers will be included and if there are any disagreements which cannot be resolved this will be brought to the third reviewer for a resolution. Any studies where there are discrepancies we will contact the authors to clarify any queries. The selection process will be documented through a PRISMA flow diagram.

Participant or population: Adults (>18 yrs) with clinically diagnosed musculoskeletal disorders completing generic or disease specific PROM(s) and eliciting TechROs, typically though the use of wearables.

Intervention: Co-administration of patient reported and technology reported outcome measures for patients with musculoskeletal conditions.

Comparator: Not applicable.

Study designs to be included: Peerreviewed primary studies and systematic literature reviews.

Eligibility criteria: 1) Peer-reviewed primary studies and literature reviews. Grey literature not included. 2) Studies which include co-administration of PatientReported Outcomes (PROMs) AND wearable electronic devices (e.g. fitness trackers, accelerometers, gyroscopes, pedometers smartphones, smartwatches) in musculoskeletal manifestations of joint pathology. Studies are EXCLUDED which 
feature wearable electronic devices but not concomitant/real time capturing of PROMs (e.g. they are recorded retrospectively/ at different timepoints). 3) Studies in languages other than English will be excluded unless a translation is available.

Information sources: MEDLINE, Embase, PsycINFO, CINAHL, the Cochrane Database of Systematic Reviews (CDSR).

Main outcome(s): Co-administration of patient reported and technology reported outcome measures for patients with musculoskeletal conditions.

Quality assessment / Risk of bias analysis: Two researchers will perform the assessments independently using a modified version of the JBI Critical Appraisal Tools, modified from checklists for quasi-experimental studies, analytical cross-sectional studies and qualitative research (https://jbi.global/criticalappraisal-tools). Any disagreement will be resolved by discussion and if necessary consultation with a third reviewer.

Strategy of data synthesis: Data will be extracted from included studies using a standardised proforma by a single reviewer and subsequently checked by a second reviewer for quality and consistency. Information on key study features such as the combined use of PROMs and TechROs will be summarised thematically. A narrative synthesis of the evidence will be conducted. The framework proposed by Rodgers (2009) will be adapted to guide this process.

Subgroup analysis: Not applicable.

Sensitivity analysis: Not applicable.

Country(ies) involved: United Kingdom.

Keywords: Musculoskeletal; Patient Reported Outcome Measures; Technology Reported Outcomes; Digital Health Technology Tool; Wearable electronic devices.
Contributions of each author:

Author 1 - Jon Evans - Will conduct title and abstract screening, data extraction, quality assessment, interpretation of findings and drafting of manuscript.

Email: j.p.evans2@exeter.ac.uk

Author 2 - lan Porter - Will devise and implement the search strategy, conduct title and abstract screening, data extraction, quality assessment interpretation of findings and drafting of manuscript.

Email: i.porter@exeter.ac.uk

Author 3 - Emma Cockcroft - Will be involved in the interpretation of findings and drafting of manuscript.

Email: e.j.cockcroft@exeter.ac.uk

Author 4 - Al-Amin Kassam - Will be involved in the interpretation of findings and drafting of manuscript.

Email: al-amin.kassam@nhs.net

Author 5 - Jose Valderas - Will contribute to title and abstract screening, data extraction, quality assessment, interpretation of findings, and drafting of manuscript.

Email: j.m.valderas@exeter.ac.uk 